\title{
Duelo Amoroso, Dependencia Emocional y Salud Mental en mujeres que han terminado una relación de pareja ${ }^{7}$
}

Jorge Arturo Martínez Gómez

PhD. En Psicología

Universidad Pedagógica y Tecnológica de Colombia Correo electrónico: jorgearturo.martinez@uptc.edu.co

Mónica Sandoval Cano

Psicóloga

Especialista en Seguridad y Salud en el Trabajo Universidad Pedagógica y Tecnológica de Colombia Correo electrónico: monica.sandoval01@uptc.edu.co

\section{Mayuris Liseth Soler Cantillo}

Psicóloga

Magister (c) en Derechos Humanos Universidad Pedagógica y Tecnológica de Colombia

Correo electrónico: mayuris.soler@uptc.edu.co

Yolima Bolívar Suárez

Magíster en Enfermería Universidad Pedagógica y Tecnológica de Colombia

\section{Resumen}

Se examinó la relación existente entre duelo amoroso, dependencia emocional y salud mental en mujeres que han terminado su relación de pareja en los últimos seis meses, mediante un diseño descriptivo-comparativo de corte transversal, en el que participaron 236 mujeres heterosexuales con edades entre los 18 y 28 años. Se utilizó el Cuestionario de Dependencia Emocional, la Escala Tridimensional de Duelo Romántico y la Lista De Verificación de Síntomas - 90-Revisado. Los resultados señalan que las emociones, tanto positivas como negativas, que experimentan las mujeres al momento de tener una ruptura amorosa, dependen de variables como la dependencia emocional y la duración de la relación; además, la ruptura y las emociones negativas podrían hacer que aparezcan síntomas de: depresión, ansiedad, hostilidad y problemas somáticos. Por lo tanto, es importante intervenir oportunamente durante el duelo romántico, para evitar que se evolucione hacia cuadros clínicos que puedan llevar a la depresión, ansiedad, pensamientos o intentos de suicidio ocasionados por el dolor de una ruptura amorosa.

Palabras clave

Duelo Amoroso; Dependencia Emocional; Salud Mental; Emociones.

7 Para citar este artículo: Martínez-Gómez, J.A., Cano-Sandoval, M., Soler-Cantillo, M. L. y Bolívar-Suárez, Y. (2021). Duelo Amoroso, Dependencia Emocional y Salud Mental en mujeres que han terminado una relación de pareja. Informes Psicológicos, 21(1), 101-116 http://dx.doi.org/10.18566/infpsic.v21n1a07 


\section{Loving grief, emotional dependence and mental health in women who have ended a relationship}

Abstract

The relationship between love grief, emotional dependence and mental health in women who have ended their relationship in the last six months was examined by means of a descriptive-comparative cross-sectional design, in which 236 heterosexual women participated with ages between the 18 and 28 years old. The Emotional Dependence Questionnaire, the Three-Dimensional Scale of Romantic Grief and the Symptom Check List - 90-Revised were used. The results indicate that the emotions, both positive and negative, that women experience when having a love breakup depend on variables such as emotional dependence and the duration of the relationship. In addition, the breakup and negative emotions could cause symptoms of depression, anxiety, hostility and somatic problems to appear. Therefore, it is important to intervene promptly during romantic grief, to prevent it from evolving into clinical conditions that can lead to depression, anxiety, thoughts or suicide attempts caused by the pain of a breakup.

Keywords Love grief, emotional dependence, mental health, emotions.

\section{Luto amoroso, dependência emocional e saúde mental em mulheres que terminaram um relacionamento \\ Resumo}

A relação entre luto amoroso, dependência emocional e saúde mental foi examinada em mulheres que encerraram 0 relacionamento nos últimos seis meses, por meio de um desenho transversal descritivo-comparativo, no qual 236 mulheres heterossexuais com idades entre os 18 e 28 anos. Foram utilizados o Questionário de Dependência Emocional, a Escala Tridimensional do Luto Romântico e a Lista de Verificação de Sintomas - 90-Revisado. Os resultados indicam que as emoções, positivas e negativas, que as mulheres vivenciam ao romperem 0 amor dependem de variáveis como a dependência emocional e a duração do relacionamento. Além disso, a separação e as emoções negativas podem fazer com que apareçam sintomas de: depressão, ansiedade, hostilidade e problemas somáticos. Portanto, é importante intervir prontamente durante o luto romântico, para evitar que evolua para condições clínicas que possam levar à depressão, ansiedade, pensamentos ou tentativas de suicídio causados pela dor de uma separação amorosa. 


\section{ntroducción}

En la actualidad la relación de pareja hace parte fundamental de la vida del ser humano y la ruptura de la misma en muchas ocasiones es un suceso inevitable que provoca un proceso de duelo que puede tener efectos en la salud física y mental de la persona (Barajas \& Cruz, 2017); por lo tanto, cada vez se hace más necesario realizar estudios que permitan identificar las emociones que experimentan las personas durante el proceso de duelo amoroso, la influencia de la dependencia emocional en éste y la afectación en la salud mental de las personas.

La relación amorosa o de pareja es un proceso de construcción social propio de la naturaleza humana, donde los sentimientos de amor son un componente esencial. El amor es considerado como fuente de inclinación emocional hacia una persona y se distinguen dos tipos: amor pasional o romántico, caracterizado por la incondicionalidad entre dos partes, además de un deseo de encuentro y de felicidad para siempre; y el amor de amistad o compañero, cuya visión de futuro se orienta a la reproducción (Rocha, Avendaño, Barrios \& Polo, 2017).

De acuerdo con Zapata y Gutiérrez (2016), desde la infancia el ser humano se encuentra en una búsqueda constante por establecer vínculos afectivos que respondan a una serie de necesidades; así, a medida que pasa el tiempo, se buscan formar relaciones de pareja que brinden seguridad, protección y estabilidad. Sin embargo, cuando estas necesidades no son cubiertas o son cubiertas de manera inestable, la dependencia emocional se puede confundir con el amor en la relación de pareja.

La dependencia emocional es vista como un tipo de dependencia sentimental que se caracteriza por la manifestación de interdependencia en las relaciones (De la Villa, Sirvent, Ovejero \& Cuetos, 2018). Como señala Urbiola y Estévez (2015), una característica esencial del ser humano es la necesidad de establecer vínculos fuertes y duraderos con el otro, por lo tanto, mientras mayor sea la importancia que las personas le otorguen a una relación de pareja, mayor será la probabilidad de caer en una dependencia emocional excesiva (Laca \& Mejía, 2017).

De esta manera, si la relación de pareja se encuentra permeada por variables como la dependencia emocional excesiva, esto puede incrementar la posibilidad de que, cuando en la relación se presente una ruptura amorosa que no se esperaba o en la que no se esté de acuerdo, se afecte el bienestar de la persona entrando en un proceso de duelo amoroso.

Según Goicoechea (2018), el proceso de duelo tiene diversas fases, pues no ocurre de manera lineal ya que pueden darse avances y retrocesos. En primer lugar, se presenta la negación de la ruptura, lo que puede llevar a la persona a querer mantener el vínculo y a la no aceptación de los límites que el otro le ha marcado; en segundo lugar, se experimentan sentimientos de rabia, que es el primer paso para aceptar la pérdida debido a que surge enojo con quien ha 
decidido romper el vínculo; en tercer lugar, se pasa a un sentimiento de tristeza, cuando se empieza a aceptar la ruptura del vínculo y, finalmente, la fase de aceptación.

El proceso de duelo puede variar de acuerdo a la historia de cada persona y el ambiente en el que se desarrolla, dependiendo de la capacidad de afrontamiento ante la pérdida, rasgos de personalidad, creencias, concepciones culturales, religiosas o la manera de ver el mundo (Uribe \& Vélez, 2016).

Las personas en el proceso de duelo experimentan emociones positivas y/o negativas, así como pueden o no utilizar estrategias de afrontamiento frente al mismo. Se ha identificado que las personas que emplean estrategias de afrontamiento como buscar apoyo social o profesional, explorar formas de solución del problema, identificar aspectos positivos de la situación o realizar actividades de esparcimiento y distracción, contribuyen a mejorar la percepción de bienestar; por el contrario, estrategias de no afrontamiento como adjudicarse la culpa, evadir o guardar para sí el problema, conllevan al deterioro de la salud mental (Sierra, Salinas \& Torres-Torija, 2017).

Otro factor de interés dentro de los estudios de ruptura y duelo amoroso, es la diferencia entre el sexo; un estudio realizado con adolescentes y adultos jóvenes encontró una diferencia importante sobre el sexo, relacionada a los pensamientos y emociones; en cuanto al pensamiento, las mujeres mostraron un mayor número de disonancias cognitivas categorizadas como extremismo y prejuicio respecto a los hombres. Para la variable emociones se encontró que las mujeres tienden a utilizar la expresión verbal como la manera de sentirse escuchada y descargar sus emociones, sub categoría que no fue identificada en los hombres (García, 2014).

Plunkett (2016) corrobora que en mujeres el impacto de la ruptura amorosa es más fuerte y que el motivo de ruptura que más genera impacto es la infidelidad, especialmente cuando existieron mayores niveles de interconexión entre la pareja. El estudio determinó que las consecuencias más importantes se relacionan con cambios en auto percepción.

Por otro lado, Sierra et al. (2017) plantearon que esta diferencia entre el sexo, no necesariamente puede relacionarse con el mayor o menor impacto psicológico entre hombres y mujeres, sino que puede deberse a aspectos sociales y culturales que privan al hombre de expresiones emocionales relacionadas con el duelo amoroso; en este sentido, las mujeres tienden a demostrar sus emociones y los hombres tienden a reprimirlas aun cuando el impacto pueda ser el mismo.

La ruptura de una relación amorosa genera desequilibrio en el ajuste emocional, siendo las emociones de sesgo depresivo, ira, enojo y de confusión las que se presentan con mayor frecuencia; además, puede verse afectada la salud física de las personas que se encuentran en un duelo amoroso (González, 2016). Por lo tanto, el objetivo de este trabajo consistió en estudiar la relación existente entre el duelo amoroso, la dependencia emocional y la salud mental, en mujeres de 18 a 28 años que han terminado una relación de pareja en los últimos seis meses. 


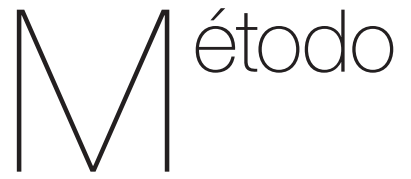

\section{Diseño}

Este trabajo utilizó un diseño observacional, de corte trasversal correlacional (Hernández, Fernández, \& Baptista, 2010), para analizar la relación que existe entre las variables duelo amoroso, dependencia emocional y salud mental en mujeres que han terminado su relación de pareja en los últimos seis meses.

\section{Participantes}

Los participantes fueron 236 mujeres heterosexuales con edades entre los $18 \mathrm{y}$ 28 años ( $M=22$; $D T=5$ años). El 72.9\% tenían estudios universitarios, un 15.3\% estudios de secundaria y el $6.8 \%$ nivel técnico de formación. El nivel socioeconómico tres fue el de mayor prevalencia con un 57.8\%; seguido del estrato dos con un 34.8\%. Todas las participantes habían terminado su relación de pareja en los últimos seis meses y no tenían una relación actual. La participación fue voluntaria, se contactaron por redes sociales y correos electrónicos para responder las pruebas, las cuales estaban programadas en Google Forms. En total contestaron 276 personas, sin embargo, se descartaron 40 cuestionarios porque algunos reportaron en la variable sexo como masculino y otros señalaron que tenía una relación al momento de diligenciar las pruebas.

\section{Instrumento}

Se diseñó una encuesta que permitió recolectar información sociodemográfica de los participantes y variables como: edad, sexo, nivel económico y nivel de formación académica; además, se preguntó por: ¿Cuánto duró su relación (meses)? y ¿Cuánto hace que terminó su relación de pareja (meses)?

\section{Cuestionario de Dependencia Emocional (Lemos-Hoyos \& Londoño-Arredondo, 2006):}

Está compuesto por 23 reactivos con seis opciones de respuesta tipo Likert, confiabilidad total por Alpha de Cronbach de .92 y se agrupa en seis sub-escalas que explican el $64.7 \%$ de la varianza así: (a) ansiedad de separación, con siete ítems y un Alpha de Cronbach de .87 que explica un 38.86\% de la varianza; (b) expresión afectiva de la pareja, compuesta por cuatro ítems, el Alpha de Cronbach de .83 y con un porcentaje de explicación de la varianza de 6.34\%; (c) modificación de planes, conformada por cuatro ítems, con Alpha de Cronbach de .75 y un 5.70\% de la varianza; (d) miedo a la soledad, que cuenta con tres ítems, Alpha de Cronbach de .79 y un $5.02 \%$ de la varianza; (e) expresión límite, con tres ítems, Alpha de Cronbach de .61 y un $4.68 \%$ de la varianza; $y$, finalmente, (f) búsqueda de atención, conformado por dos ítems, con un Alpha de Cronbach de .77 y con un $4.10 \%$ de la varianza. En el estudio de Lemos \& Londoño (2006), la muestra estuvo conformada por 815 participantes del Área metropolitana de Medellín - Colombia, con edades entre los 16 y los 55 años, distribuidos por sexo con 506 mujeres (62.1\%) y 309 hombres (37.9\%). 
Escala tridimensional de duelo romántico (Sánchez-Aragón \& Retana-Franco, 2013):

Está compuesto por 164 reactivos, con cinco opciones de respuesta tipo Likert que indican el grado de acuerdo ante cada afirmación del instrumento, agrupados en tres factores de orden superior: (a) emociones, constituida por cuatro sub-factores: Emociones negativas, emociones positivas, esperanza y decepción. Esta subescala explica el 59.56\% de la varianza y tiene coeficientes Alpha de Cronbach entre .61 y .96; (b) pensamientos, que consta de seis sub-factores: Anhelo, negociación, reacciones hostiles hacia la ex pareja, suspicacia, aceptación, reorganización y sustitución, con un Alpha de Cronbach que oscila entre .61 y .93; (c) conductas ante la experiencia de duelo por rompimiento amoroso, con seis sub-factores: re-adaptación, reacciones hostiles hacia otros, depresión, desorganización del sí mismo, negación y agresión, con un Alpha de Cronbach que oscila entre .88 a .94 . En el estudio de Sánchez-Aragón y Retana-Franco (2013) se utilizó una muestra no probabilística de 833 participantes de la Ciudad de Méxi$\mathrm{co}$, con edades comprendidas entre los 17 y 56 años.

\section{Lista de Verificación de Síntomas - 90- Revisado (Casullo \& Pérez, 2008):}

Este instrumento fue modificado a partir del El SCL-90 (lista de chequeo de síntomas-90), el cual fue diseñado en la Universidad Johns Hopkins para la evaluación de un espectro de dimensiones de psicopatología, tanto en pacientes psiquiátricos como en población normal.
El SCL-90-R es la versión del cuestionario desarrollada y reformada por Derogatis (1994), donde se ajustaron de manera parcial siete ítems y se sustituyeron dos. Esta lista evalúa la angustia psicológica en términos de nueve dimensiones de síntomas primarios: somatización (12 ítems), Obsesividad-compulsividad (10 ítems), Sensibilidad interpersonal (9 ítems), Depresión (13 ítems), Ansiedad (10 ítems), Hostilidad (6 ítems), Ansiedad fóbica (7 ítems), Ideación paranoide (6 ítems) y Psicoticismo (10 ítems). Incluye también siete dimensiones adicionales que constan de 1 ítem cada una (pérdida del apetito, aumento del apetito, insomnio de conciliación, alteraciones en el mantenimiento del sueño, despertar prematuro, sentimientos de culpa y pensamientos de muerte). Finalmente proporciona tres índices globales (índice de sintomatología general -GSI-, índice de alteración de los síntomas positivos -PSDI- y el total de síntomas positivos -PST), que permiten analizar al evaluado en tres niveles de complejidad (global, dimensional y sintomático).

Este instrumento ha sido validado en diversos países y poblaciones; para la presente investigación se tendrán en cuenta los resultados de Caparrós et al. (2007), que analizaron las propiedades psicométricas de esta escala en una muestra de 1.277 estudiantes de la Universidad de Girona- España. La consistencia interna por Alfa de Cronbach para las nueve dimensiones primarias y el GSI fue: Somatización (.84), Obsesividadcompulsividad (.80), Sensibilidad interpersonal (.82), Depresión (.88), Ansiedad (.83), Hostilidad (.76), Ansiedad fóbica (.69), Ideación paranoide (.74), Psicoticismo (.75) y GSI (.97). 


\section{Procedimiento}

Se programaron las pruebas en Google Forms; después por redes sociales se realizó una convocatoria donde se explicó el objetivo, características y los criterios que debían tener las personas que querían participar en la investigación. En las redes sociales se dejó el enlace para contestar el cuestionario y en algunos casos se envió por correo electrónico, a mujeres que accedían a participar en la investigación. Para poder contestar los cuestionarios, los participantes debían leer el consentimiento y aceptar la participación en el estudio de forma voluntaria, la cual fue anónima y no remunerada. Sin embargo, los participantes tenían la opción de dejar como contacto el correo electrónico, para recibir retroalimentación de los resultados de la investigación y, de forma particular, el análisis de sus resultados en el estudio. En promedio, los cuestionarios fueron contestados en 15 minutos y la información quedaba en una base de datos de Excel, la cual se exportó a SPSS 21.0 para hacer los análisis estadísticos.

\section{Análisis estadístico}

Se utilizó el programa SPSS versión 21.0 para realizar los análisis estadísticos. Para caracterizar a los participantes se emplearon descriptivos mediante frecuencias, porcentajes y, para las variables edad, duración de la relación y tiempo trascurrido desde la finalización de la relación, analizándose mediante medidas de tendencia central (media) y de dispersión (DS). Se asumió que los datos tenían una distribución libre, debido a que la prueba de normalidad Shapiro-Wilk mostró que algunas variables no se ajustaban normalmente $(p \leq .05)$. Para efectuar las correlaciones, se utilizó el coeficiente Rho de Spearman.

\section{Consideraciones éticas}

La investigación tenía un nivel de riesgo mínimo (Resolución 008430 de 1993); se solicitó el consentimiento informado para la participación, donde se les informó objetivos, procedimientos, posibles consecuencias y la relevancia social-científica del trabajo; además, se señalaron los derechos de confidencialidad y anonimato de la participación. Los principios éticos estaban enmarcados en la Ley 1090 del 6 de septiembre de 2006, que reglamenta el ejercicio de la psicología, mediante la responsabilidad, derecho a la no-participación, el respeto, confidencialidad de la información recogida y derecho a ser informado de los resultados, además de evitar el uso indebido de los datos recolectados.

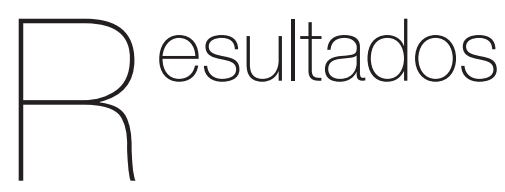

Para cumplir con el objetivo propuesto en este estudio, primero se realizó una correlación entre las emociones que mide el instrumento tridimensional de duelo romántico (Dolor, Confusión, Depresión, Desesperación, Debilidad, Ansiedad, Desánimo, Soledad, Melancolía, Enojo, Tristeza, Falta de motivación, Miedo, Ira Inseguridad, Ambivalencia, Incertidumbre, Ilusión, Nostalgia, Ternura, Alegría, 
Desahogo, Gusto, Alivio, Bienestar, Calma, Ánimo, Paz, Felicidad, Optimismo, Tranquilidad, Euforia, Entusiasmo, Gozo, Relajación, Resignación y Superación) y las variables dependencia emocional y salud mental (ver tablas 1, 2, 3 y 4). En segundo lugar, se procedió a correlacionar los factores del instrumento tridimensional de duelo romántico, los factores de los instrumentos Dependencia Emocional y Lista de Verificación de Síntomas - 90 con las siguientes preguntas: (a) ¿Cuánto duró su relación? (meses) y (b) ¿Cuánto hace que terminó su relación de pareja? (meses) (ver Tabla 5).

Tabla 1

Correlación entre las variables emociones negativas, dependencia emocional y salud mental en 236 mujeres.

\begin{tabular}{|c|c|c|c|c|c|c|c|c|c|c|}
\hline \multirow[b]{2}{*}{$\begin{array}{l}\text { DUELO ROMANTICO- } \\
\text { EMOCIONES }\end{array}$} & \multicolumn{6}{|c|}{ FACTORES: DEPENDENCIA EMOCIONAL } & \multicolumn{4}{|c|}{ FACTORES: SALUD MENTAL } \\
\hline & 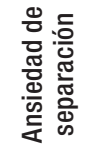 & 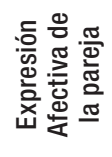 & 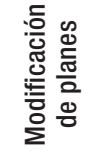 & 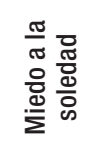 & 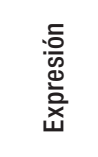 & 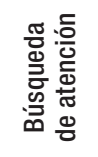 & 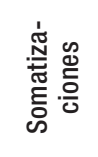 & 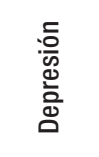 & 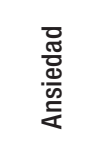 & 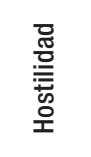 \\
\hline Dolor & $.409^{\star \star}$ & $.225^{\star \star}$ & $.281^{\star \star}$ & $.180^{\star *}$ & $.200^{\star \star}$ & -.005 & $.414^{\star \star}$ & $.470^{\star \star}$ & $.380^{\star \star}$ & $.348^{\star \star}$ \\
\hline Confusión & $.311^{\star *}$ & .086 & $.281^{\star *}$ & $.225^{\star *}$ & $.203^{\star \star}$ & -.051 & $.444^{\star *}$ & $.481^{\star *}$ & $.458^{\star \star}$ & $.302^{\star *}$ \\
\hline Depresión & $.383^{\star \star}$ & $.280^{\star \star}$ & $.299^{\star *}$ & $.376^{\star \star}$ & $.433^{\star \star}$ & .056 & $.452^{\star *}$ & $.533^{\star \star}$ & $.487^{\star *}$ & $.316^{\star \star}$ \\
\hline Desesperación & $.472^{\star \star}$ & $.355^{\star \star}$ & $.333^{\star \star}$ & $.385^{\star \star}$ & $.500^{\star \star}$ & .039 & $.537^{\star *}$ & $.611^{\star *}$ & $.539^{\star \star}$ & $.401^{\star \star}$ \\
\hline Debilidad & $.451^{\star \star}$ & $.226^{\star \star}$ & $.294^{\star *}$ & $.341^{\star *}$ & $.467^{\star *}$ & .038 & $.397^{\star *}$ & $.471^{\star \star}$ & $.498^{\star *}$ & $.411^{\star *}$ \\
\hline Ansiedad & $.408^{\star \star}$ & $.420^{\star \star}$ & $.469^{\star \star}$ & $.418^{\star \star}$ & $.440^{\star \star}$ & $.190^{\star \star}$ & $.403^{\star \star}$ & $.487^{\star \star}$ & $.554^{\star \star}$ & $.480^{\star \star}$ \\
\hline Desanimo & $.530^{\star \star}$ & $.273^{\star \star}$ & $.340^{\star *}$ & $.261^{\star *}$ & $.347^{\star *}$ & .085 & $.508^{\star *}$ & $.491^{\star *}$ & $.454^{\star *}$ & $.424^{\star *}$ \\
\hline Soledad & $.382^{\star \star}$ & $.152^{\star}$ & $.259^{\star \star}$ & $.278^{\star \star}$ & $.177^{\star \star}$ & .003 & $.539^{\star \star}$ & $.527^{\star \star}$ & $.420^{\star \star}$ & $.433^{\star \star}$ \\
\hline Melancolía & $.398^{\star \star}$ & $.291^{\star \star}$ & $.281^{\star *}$ & $.294^{\star \star}$ & $.449^{\star \star}$ & $.128^{\star}$ & $.517^{\star *}$ & $.521^{\star \star}$ & $.506^{\star \star}$ & $.411^{\star \star}$ \\
\hline Enojo & $.350^{* *}$ & $.268^{\star \star}$ & $.341^{\star *}$ & $.273^{\star \star}$ & $.215^{\star \star}$ & .030 & $.390^{\star *}$ & $.436^{\star \star}$ & $.435^{\star *}$ & $.482^{\star \star}$ \\
\hline Tristeza & $.474^{\star \star}$ & $.287^{* \star}$ & $.403^{\star \star}$ & $.317^{\star \star}$ & $.218^{\star *}$ & .084 & $.426^{\star *}$ & $.519^{\star \star}$ & $.464^{\star *}$ & $.324^{\star \star}$ \\
\hline Falta de motivación & $.440^{\star \star}$ & $.162^{*}$ & $.219^{\star \star}$ & $.332^{\star *}$ & $.229^{\star *}$ & .081 & $.544^{\star \star}$ & $.458^{\star \star}$ & $.427^{\star *}$ & $.388^{\star *}$ \\
\hline Miedo & $.435^{\star \star}$ & $.182^{\star \star}$ & $.194^{\star \star}$ & $.369^{\star \star}$ & $.292^{\star \star}$ & .103 & $.447^{\star \star}$ & $.478^{\star \star}$ & $.471^{\star *}$ & $.423^{\star \star}$ \\
\hline Ira & $.351^{* *}$ & $.238^{\star \star}$ & $.259^{\star \star}$ & $.289^{\star \star}$ & $.313^{\star *}$ & .011 & $.343^{\star *}$ & $.424^{\star \star}$ & $.378^{\star *}$ & $.424^{\star *}$ \\
\hline Inseguridad & $.471^{\star \star}$ & $.158^{\star}$ & $.223^{\star *}$ & $.271^{\star *}$ & $.351^{* \star}$ & .045 & $.534^{\star *}$ & $.512^{\star \star}$ & $.528^{\star \star}$ & $.483^{\star *}$ \\
\hline
\end{tabular}

Nota: en negrita se muestran las correlaciones significativas. ${ }^{\star} p<.05,{ }^{* *} p<.01,{ }^{* \star *} p<.001$

Se encontró una correlación significativa entre 42 de las 43 emociones evaluadas con la escala de emociones del duelo romántico y los seis factores (ansiedad de separación, expresión afectiva de la pareja, modificación de planes, miedo a la soledad, expresión, búsqueda de atención) que mide la escala de dependencia emocional (ver tablas 1, 2, 3 y 4). La ansiedad de separación correlacionó con 42 de las 43 emociones evaluadas, la expresión afectiva de pareja correlacionó con 30 de las 43 emociones evaluadas, la modificación de planes 
con 41 de las 43 emociones evaluadas, el miedo a la soledad con 32 de las 43 emociones evaluadas, la expresión límite con 33 de las 43 emociones evaluadas y, finalmente, la búsqueda de atención con 7 de las 43 emociones evaluadas (ver tablas 1, 2, 3 y 4).

Tabla 2

Correlación entre las variables emociones positivas, dependencia emocional y salud mental en 236 mujeres.

\begin{tabular}{|c|c|c|c|c|c|c|c|c|c|c|}
\hline \multirow[b]{2}{*}{$\begin{array}{l}\text { DUELO } \\
\text { ROMANTICO-EMOCIONES }\end{array}$} & \multicolumn{6}{|c|}{ FACTORES: DEPENDENCIA EMOCIONAL } & \multicolumn{4}{|c|}{ FACTORES: SALUD MENTAL } \\
\hline & 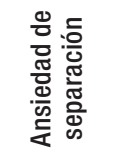 & 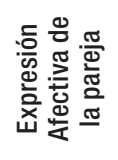 & 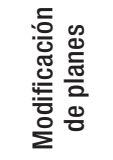 & 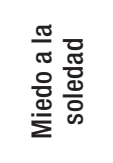 & 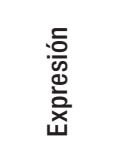 & 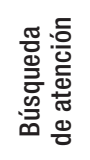 & 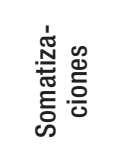 & $\begin{array}{l}: \text { 흥 } \\
\text { 흘 } \\
\text { 흄 }\end{array}$ & $\begin{array}{l}\text { 웡 } \\
\frac{d}{6} \\
\frac{\pi}{4}\end{array}$ & $\begin{array}{l}\text { 흃 } \\
\text { 을 } \\
\text { 오 }\end{array}$ \\
\hline Alegría & $-.227^{\star \star}$ & -.051 & $-.170^{\star *}$ & -.027 & -.023 & .127 & $-.224^{\star \star}$ & $-.201^{\star \star}$ & -.108 & -.032 \\
\hline Desahogo & $-.215^{\star \star}$ & .051 & $-.201^{\star \star}$ & -.030 & -.065 & .070 & $-.235^{\star \star}$ & $-.226^{\star \star}$ & $-.163^{*}$ & .088 \\
\hline Gusto & $-.271^{\star \star}$ & $-.132^{\star}$ & $-.226^{\star \star}$ & -.018 & -.042 & .103 & $-.346^{\star \star}$ & $-.403^{\star *}$ & $-.253^{\star \star}$ & -.108 \\
\hline Alivio & $-.161^{*}$ & -.010 & $-.133^{\star}$ & $.182^{\star \star}$ & -.006 & .083 & -.109 & -.107 & -.057 & .122 \\
\hline Bienestar & $-.161^{*}$ & -.121 & $-.183^{\star \star}$ & .040 & -.001 & .116 & $-.333^{\star \star}$ & $-.354^{\star \star}$ & $-.240^{\star \star}$ & -.106 \\
\hline Calma & $-.340^{\star *}$ & $-.231^{* *}$ & $-.373^{\star \star}$ & -.088 & $-.235^{\star \star}$ & .020 & $-.553^{\star \star}$ & $-.549 \star *$ & $-.391^{\star \star}$ & $-.355^{\star \star}$ \\
\hline Animo & $-.343^{\star \star}$ & -.064 & $-.269^{\star \star}$ & -.090 & -.095 & .096 & $-.422^{\star \star}$ & $-.429 \star \star$ & $-.331^{\star \star}$ & $-.156^{\star}$ \\
\hline Paz & $-.348^{\star \star}$ & $-.151^{*}$ & $-.265^{\star \star}$ & -.055 & $-.136^{*}$ & .007 & $-.397^{\star \star}$ & $-.410^{\star \star}$ & $-.379^{\star \star}$ & $-.169^{\star *}$ \\
\hline Felicidad & $-.217^{\star \star}$ & $-.140^{*}$ & $-.259^{\star \star}$ & $-.182^{\star *}$ & .009 & .041 & $-.352^{\star \star}$ & $-.355^{\star \star}$ & $-.306^{\star \star}$ & $-.234^{\star *}$ \\
\hline Optimismo & -.108 & -.035 & $-.143^{\star}$ & -.010 & $-.136^{\star}$ & .089 & $-.296^{\star *}$ & $-.368^{\star \star}$ & $-.238^{\star \star *}$ & -.088 \\
\hline Tranquilidad & $-.350^{\star *}$ & $-.241^{\star \star}$ & $-.298^{\star \star}$ & -.047 & $-.180^{* *}$ & -.084 & $-.406^{\star \star}$ & $-.436^{\star \star}$ & $-.360^{\star \star}$ & $-.221^{\star \star}$ \\
\hline Euforia & $-.250^{\star \star}$ & -.063 & $-.264^{\star \star}$ & -.065 & $-.136^{\star}$ & .054 & $-.345^{\star \star}$ & $-.362^{\star \star}$ & $-.314^{\star \star}$ & $-.253^{\star \star}$ \\
\hline Entusiasmo & $-.288^{\star \star}$ & $-.170^{\star *}$ & $-.267^{\star \star}$ & $-.269^{\star \star *}$ & $-.186^{\star *}$ & .049 & $-.413^{\star \star}$ & $-.454^{\star \star}$ & $-.316^{\star \star}$ & $-.272^{\star \star}$ \\
\hline Gozo & $-.307^{\star \star}$ & $-.205^{\star \star}$ & $-.343^{\star \star}$ & $-.264^{\star \star}$ & $-.181^{\star *}$ & .042 & $-.396^{\star \star}$ & $-.473^{\star \star}$ & $-.362^{\star \star}$ & $-.176^{\star \star}$ \\
\hline Relajación & $-.345^{\star \star}$ & $-.199^{\star \star}$ & $-.216^{\star \star}$ & -.068 & $-.140^{*}$ & -.116 & $-.264^{\star \star}$ & $-.276^{\star \star}$ & $-.260^{\star *}$ & $-.141^{*}$ \\
\hline Resignación & $.161^{*}$ & .025 & $.147^{\star}$ & $.292^{\star \star}$ & .009 & $.165^{\star}$ & $.272^{\star \star}$ & $.172^{\star \star}$ & $.266^{\star \star}$ & .087 \\
\hline Superación & $-.382^{\star *}$ & -.106 & $-.351^{\star \star}$ & $-.238^{\star \star}$ & $-.410^{\star *}$ & -.031 & $-.344^{\star \star}$ & $-.414^{\star *}$ & $-.311^{\star *}$ & $-.326^{\star \star}$ \\
\hline
\end{tabular}

Nota: en negrita se muestran las correlaciones significativas. ${ }^{\star} \mathrm{p}<.05,{ }^{\star \star} \mathrm{p}<.01,{ }^{\star \star \star} \mathrm{p}<.001$

La ansiedad de separación, la expresión afectiva de la pareja, la modificación de planes, el miedo a la soledad, la expresión y la búsqueda de atención, obtuvieron una correlación positiva con las emociones negativas; de esta forma, si se obtienen puntuaciones altas en los factores que miden la escala de dependencia emocional, las emociones más frecuentes serían: decepción, coraje, dolor, confusión, desilusión, ambivalencia, depresión, desesperación, debilidad, ansiedad, desánimo, soledad, impotencia, melancolía, incertidumbre, enojo, tristeza, nostalgia, frustración, incertidumbre, resignación, falta de motivación, miedo, ira e inseguridad (ver tablas 1, 2, 3 y 4). 
Tabla 3

Correlación entre las variables emociones: Decepción- esperanza, dependencia emocional y salud mental en 236 mujeres.

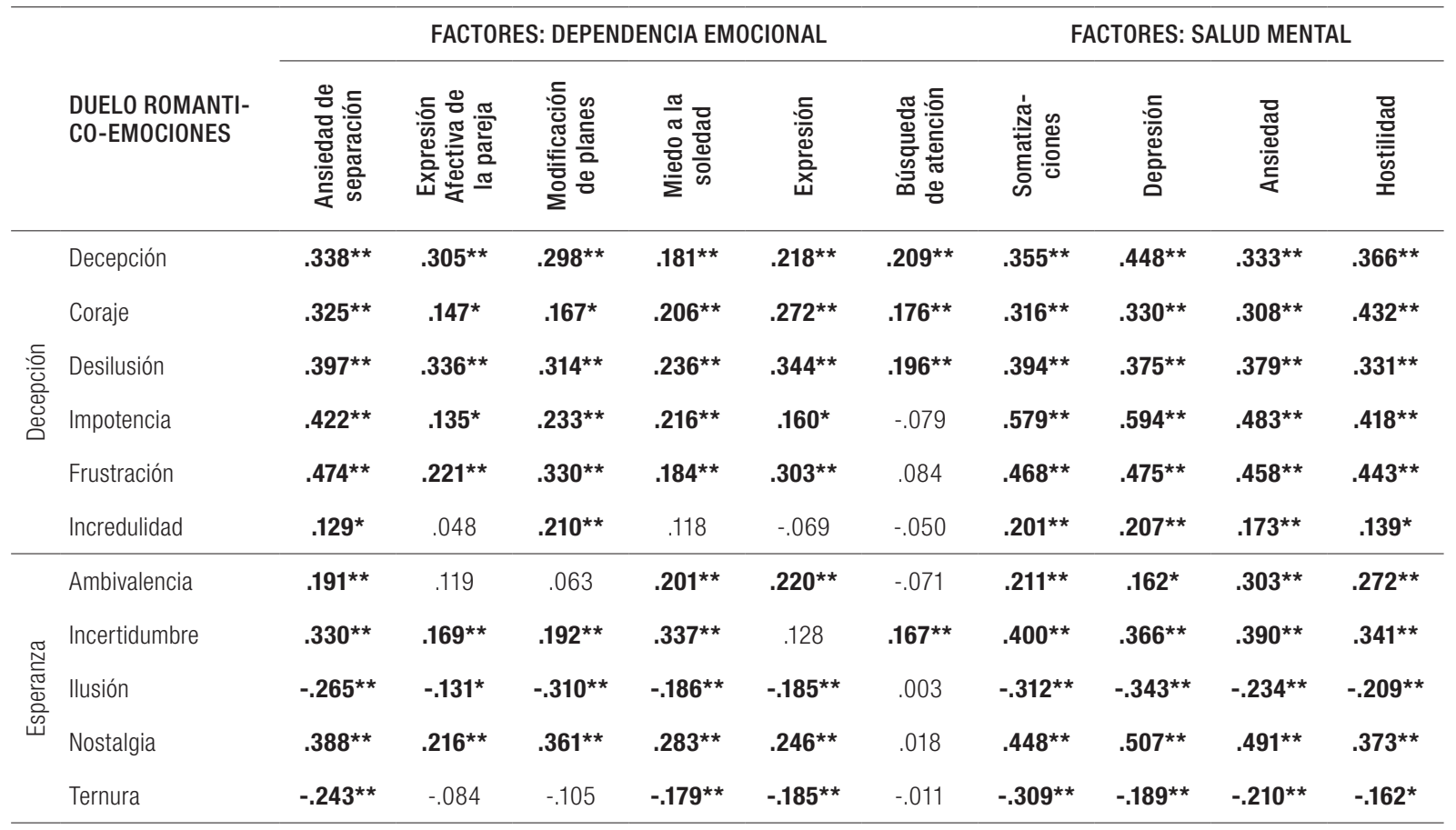

Nota: en negrita se muestran las correlaciones significativas.

${ }^{*} p<.05,{ }^{* *} p<.01,{ }^{* *} p<.001$

Además, estos seis factores que pertenecen a la escala de dependencia emocional, correlacionaron negativamente con las emociones positivas, entonces, se podría pensar que, a mayor puntuación en ansiedad de separación, expresión afectiva de la pareja, modificación de planes, miedo a la soledad y expresión, menor serán las emociones como alegría, desahogo, gusto, alivio, bienestar, calma, ánimo, paz, felicidad, euforia, entusiasmo, gozo, ilusión, ternura, relajación, superación, tranquilidad, optimismo, tranquilidad y motivación (ver tablas 1, 2 y 3).

Con relación a los factores de Salud Mental, se encontró una correlación significativa entre somatización y depresión con 42 de las 43 emociones evaluadas en este estudio. Para somatización, la mayor fuerza de relación $\left(\geq .50^{\star}\right)$ fue con las siguientes emociones: desesperación $\left(r_{s}=.537^{\star \star}\right)$, calma $\left(r_{s}=-.553^{\star \star}\right)$, desánimo $\left(r_{s}=.508^{\star \star}\right)$, soledad $\left(r_{s}=.539^{\star \star}\right)$, impotencia $\left(r_{s}=.579^{\star \star}\right)$, melancolía $\left(r_{s}=.517^{\star \star}\right)$, falta de motivación $\left(r_{s}=.544^{\star \star}\right)$ e inseguridad $\left(r_{s}=.534^{\star *}\right)$. De igual forma, con depresión, la mayor fuerza de relación $(\geq$ $\left..50^{\star}\right)$ fue con las siguientes emociones: depresión $\left(r_{s}=.533^{\star \star}\right)$, desesperación $\left(r_{s}=.611^{\star \star}\right)$, calma $\left(r_{s}=-.549^{\star \star}\right)$, soledad $\left(r_{s}=.527^{\star \star}\right)$, impotencia $\left(r_{s}=.594^{\star *}\right)$, melancolía $\left(r_{s}=.521^{\star \star}\right)$, tristeza $\left(r_{s}=.519^{\star \star}\right)$, nostalgia $\left(r_{s}=.507^{\star *}\right)$ e inseguridad $\left(r_{s}=\right.$ $\left..512^{\star \star}\right)$. En cuanto a la ansiedad, la mayor fuerza de relación $\left(\geq .50^{\star}\right)$ estuvo con las siguientes emociones: desesperación $\left(r_{s}\right.$ $\left.=.539^{\star \star}\right)$, ansiedad $\left(r_{s}=.554^{\star \star}\right)$, melancolía $\left(r_{s}=.506^{\star \star}\right)$ e inseguridad $\left(r_{s}=.528^{\star \star}\right)$. No obstante, ninguna emoción tuvo una fuerza de relación superior o igual a .500*. 
Se puede observar en las tablas 1 y 2 que los cuatro factores (somatización, depresión, ansiedad y hostilidad), correlacionaron de forma positiva con las emociones negativas, así como negativamente con las emociones positivas.

Tabla 4

Correlación entre las variables emociones, dependencia emocional y salud mental en 236 mujeres.

\begin{tabular}{|c|c|c|c|c|}
\hline \multirow{2}{*}{$\begin{array}{l}\text { Factores: Salud Mental y } \\
\text { Dependencia Emocional }\end{array}$} & \multicolumn{4}{|c|}{ Factores Duelo Romántico-Emociones } \\
\hline & Decepción & Emociones Negativas & Esperanza & Emociones Positivas \\
\hline ¿Cuánto duró su relación? (meses) & -.028 & $.222^{\star \star}$ & $-.133^{\star}$ & $-.188^{\star \star}$ \\
\hline $\begin{array}{l}\text { ¿Cuánto hace que terminó su } \\
\text { relación de pareja? (meses) }\end{array}$ & -.083 & -.078 & $.301^{\star \star}$ & $.383^{\star \star}$ \\
\hline Ansiedad de separación & $.467^{\star \star}$ & $.536^{\star \star}$ & $-.411^{\star *}$ & $-.352^{\star *}$ \\
\hline Expresión Afectiva de la pareja & $.273^{\star \star}$ & $.308^{\star \star}$ & $-.190 * \star$ & $-.176^{\star \star}$ \\
\hline Modificación de planes & $.325^{\star \star}$ & $.383^{\star \star}$ & $-.325^{\star \star}$ & $-.327^{\star \star}$ \\
\hline Miedo a la soledad & $.244^{\star \star}$ & $.381^{\star *}$ & $-.297^{\star *}$ & -.104 \\
\hline Expresión & $.295^{\star \star}$ & $.418^{\star \star}$ & $-.242^{\star \star}$ & $-.163^{\star}$ \\
\hline Búsqueda de atención & .116 & .065 & -.032 & .052 \\
\hline Somatizaciones & $.523^{\star \star}$ & $.597^{\star \star}$ & $-.501 * \star$ & $-.460 * *$ \\
\hline Depresión & $.545^{\star \star}$ & $.631^{\star \star}$ & $-.471^{\star \star}$ & $-.493^{\star \star}$ \\
\hline Ansiedad & $.482^{\star \star}$ & $.601^{\star \star}$ & $-.437^{\star \star}$ & $-.387^{\star \star}$ \\
\hline Hostilidad & $.477^{\star \star}$ & $.534^{\star \star}$ & $-.409 \star \star$ & $-.216^{\star \star}$ \\
\hline
\end{tabular}

Nota: en negrita se muestran las correlaciones significativas. ${ }^{\star} p<.05,{ }^{* \star} p<.01,{ }^{\star \star \star \star} p<.001$

En cuanto a la duración de las relaciones de pareja, se encontró una relación con las emociones negativas $\left(r_{s}=.222^{\star \star}\right)$, la esperanza $\left(r_{s}=-.133^{\star \star}\right)$ y emociones positivas $\left(r_{s}=-.188^{\star \star}\right)$. Lo anterior podría suponer que, a mayor duración de una relación, al finalizar ésta se presentarán mayores emociones negativas y menores emociones positivas y esperanza. De igual forma, el tiempo que ha trascurrido desde la terminación de la relación, se correlaciona con la esperanza $\left(r_{s}=.301^{\star \star}\right)$ y emociones positivas $\left(r_{s}=.383^{\star \star}\right)$. Esto podría presumir que, a mayor tiempo de terminación de la relación de pareja, mayores son las emociones positivas y de esperanza que aparecen en las mujeres.

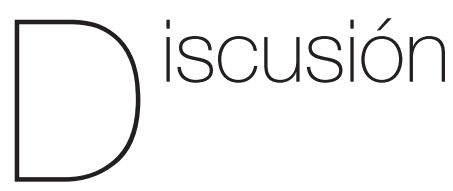

Se encontró que las emociones negativas que experimentan las mujeres al terminar una relación de pareja están relacionadas con el tipo de dependencia emocional, probablemente porque las personas que son dependientes emocionales en sus relaciones, manifiestan mayor miedo a la soledad, tienen más necesidad de afecto y sentimientos de ser queridos, presentando aumento en síntomas de depresión y ansiedad. De 
esta forma, las mujeres con dependencia emocional pueden experimentar con mayor intensidad las etapas del duelo romántico, siendo la fase de desorientación la que más desequilibrio emocional les causa, caracterizada por sentimientos negativos asociados con desesperanza que les impide continuar con la rutina diaria. Además, la fase de superación se presenta de forma más tardía donde manifiestan dificultades para sentir emociones positivas que les permitan percibir que han podido superar la ruptura amorosa.

La relación entre dependencia emocional y las emociones negativas que pueden experimentar las mujeres al terminar sus relaciones de pareja, se debe probablemente a que la dependencia emocional hace parte de un patrón de necesidades emocionales insatisfechas que se intentan satisfacer de manera desadaptativa con otras personas, en este caso con la pareja amorosa, donde se podría presentar una falta de autonomía en la vida cotidiana, inseguridad, carencia de iniciativa, dificultades para tomar decisiones, además de una búsqueda excesiva de recompensas afectivas, llevando a relaciones de pareja no sanas y desequilibradas (Castelló, 2005). Por lo tanto, la ruptura le ocasionará mayor dolor y sufrimiento, con sentimientos negativos de ansiedad, desesperanza y depresión, porque en su relación amorosa siempre busca hacer todo lo que su pareja quiere con el fin de agradarle, dándole todo tipo de atención y privilegios, muchas veces de forma sumisa.

Además, en este estudio se encontró que existe relación entre las emociones que experimentan las mujeres al terminar su relación de pareja con cuatro factores de salud mental (somatización, depresión, ansiedad y hostilidad). Por lo tanto, las mujeres que experimentan con mayor intensidad emociones negativas después de una ruptura amorosa pueden presentar síntomas psicopatológicos como depresión, ansiedad, hostilidad, a nivel físico, cuadros de somatización, lo que genera afectaciones en su salud mental. Estos resultados son acordes con el estudio desarrollado por Barajas, González, Cruz y Robles (2012) donde identificaron que, posterior a una ruptura, surgen emociones negativas que pueden afectar de manera importante a salud física y mental, presentándose con mayor intensidad angustia psicológica, desesperación, estrés, impotencia y deseo de venganza.

De acuerdo con Aiquipa (2015), cuando hubo dependencia emocional, prevalecen y se prolongan los síntomas negativos durante el duelo, pues se evidencian emociones como sentir ansiedad, depresión o incluso pensamientos suicidas lo que podría indicar una afectación importante en la salud mental del individuo y conllevar a una gran variedad de cambios de humor, que van desde la euforia, hasta la ansiedad, la desesperación e incluso la ira.

Keller y Nesse (2005) señalan que las consecuencias que se experimentan con mayor frecuencia tras la pérdida de una pareja son la tristeza y el llanto; de igual forma, Locker, Mclntosh, Hackney, Wilson y Wiegand (2010) indican el enojo, la inseguridad, celos hacia la ex pareja, disminución de la autoestima, un pobre autoconcepto, como consecuencias de las rupturas. Para Fisher (2005), las personas después de una ruptura presentan con mayor intensidad depresión y ansiedad y suicidios ocasionados por el dolor de una 
ruptura amorosa. Además, pueden aparecer conductas autodestructivas caracterizadas por autolesiones, consumo de alcohol y sustancias psicoactivas (Davis, Shaver \& Vernon, 2003). De igual forma, Tashiro y Frazier (2003) consideran que si una ruptura amorosa no se maneja adecuadamente puede llegar a tener consecuencias muy graves que van desde la tristeza y el enojo, hasta desórdenes psicológicos tales como la ansiedad y la depresión.

Finalmente, se encontró en este estudio que el tiempo que dura una relación se correlaciona con el tipo de emociones que se experimentan al momento de la ruptura, por ejemplo, cuanto más tiempo han durado juntos los miembros de la pareja, estos experimentarán más emociones negativas (dolor, confusión, depresión, desesperación, debilidad, ansiedad, desánimo, soledad, melancolía, enojo, tristeza, falta de motivación, miedo, ira e inseguridad) al momento de terminar su relación. Lo anterior probablemente se debe porque cuando una pareja tiene una mayor duración en la relación, existen más recuerdos de experiencias vividas, metas y proyectos planificados, de modo que, después de la ruptura, habrá más dificultad en evitar pensamientos negativos sobre su ex pareja si es que su rutina diaria se entrelazaba y se compartía (García, 2014).

No obstante, se encontró que a mayor tiempo que ha trascurrido desde la ruptura de su relación de pareja, se presentan más sentimientos de esperanza y más emociones positivas como alegría, desahogo, gusto, alivio, bienestar, calma, ánimo, paz, felicidad, optimismo, tranquilidad, euforia, entusiasmo, gozo, relajación, resignación y superación. Por lo tanto, se podría suponer que el tiempo que duró la relación amorosa y el tiempo que ha transcurrido desde la ruptura, son factores que pueden incidir en la sensación de sentimientos, tanto positivos como negativos, afectando la percepción de desamor y duelo romántico en las mujeres.

Lo anterior concuerda con lo que afirma Cabodevilla (2007), quien señala que la estrategia fundamental para el afrontamiento y manejo del duelo es darse tiempo para reestablecer algunos procesos psicológicos, aceptando los cambios de vida sin esa persona; en este sentido, juega un papel fundamental el tiempo y las herramientas que utilice el individuo para reconstruir su vida y generar aceptación emocional y racional frente a la ruptura. Por lo tanto, el efecto positivo o negativo que tenga durante una ruptura de pareja, dependerá de factores como qué tan importante era la relación, su duración, quién decidió romper, los motivos de ruptura, el grado de involucramiento, entre otros (Márquez, Del Castillo \& Balderrama, 2017).

En conclusión, los resultados obtenidos en este estudio, en concordancia con trabajos previos, señalan que las emociones que experimentan las mujeres al momento de tener una ruptura amorosa dependen en medida de variables como la dependencia emocional y la duración de la relación; además, la ruptura y las emociones negativas pueden hacer aparecer síntomas de depresión, ansiedad, hostilidad y problemas somáticos. Por lo tanto, es importante intervenir oportunamente durante el duelo romántico, para evitar trascender a cuadros clínicos que puedan llevar a depresión, ansiedad, pensamiento o intentos de suicidio 
ocasionados por el dolor de una ruptura amorosa, así como evitar las conductas autodestructivas caracterizadas por autolesiones, consumo de alcohol y sustancias psicoactivas.

Es importante señalar que, debido al tipo de muestreo para seleccionar a las participantes y el diseño que se utilizó, los resultados de este estudio deben ser considerados como exploratorios y su análisis debe ser cuidadoso en su generalización, pues se limitan a mujeres de un área geográfica muy específica de Colombia, que presentaron una relación heterosexual, lo que conlleva a la necesidad de realizar una investigación con una muestra más amplia con diferentes orientaciones sexuales y en otras regiones del país.

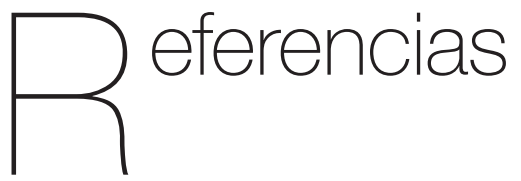

Aiquipa, T. (2015). Dependencia emocional en mujeres víctimas de violencia de pareja. Revista de Psicología, 33(2), 411-437.

Barajas, M., \& Cruz del Castillo, C. (2017). Ruptura de la pareja en jóvenes: factores relacionados con su impacto. Enseñanza e Investigación en Psicología, 22(3), 342-352.

Barajas M., González C., Cruz, C., \& Robles, R. (2012). El significado psicológico de una ruptura de pareja significativa en jóvenes universitarios. Psicología Iberoamericana, 20(2), 26-32.
Cabodevilla, I. (2007). Las pérdidas y sus duelos. Anales del sistema sanitario de Navarra, 30(3), 163-176. doi:10.23938/ ASSN.0209.

Caparrós-Caparrós, B., \& Villar-Hoz, E., Juan-Ferrer, J., \& Viñas-Poch, F. (2007). Symptom Check-List-90-R: fiabilidad, datos normativos y estructura factorial en estudiantes universitarios. International Journal of Clinical and Health Psychology, 7(3), 781-794.

Castelló, J. (2005). Dependencia Emocional. Características y Tratamiento. Madrid, España: Alianza Editorial.

Casullo, M., \& Pérez, M. (2008). El inventario de síntomas SCL-90-R de Derogatis. Buenos Aires, Argentina: Departamento de Publicaciones. Facultad de Psicología. UBA

Davis, D., Shaver, P., \& Vernon, M. (2003). Physical, emotional, and behavioral reactions to breaking up: The roles of gender, age, emotional involvement, and attachment style. Personality and Social Psychology Bulletin, 29(7), 871-884.

De la Villa, M., Sirvent, C., Ovejero, A., \& Cuetos, G. (2018). Dependencia emocional en las relaciones de pareja como Síndrome de Artemisa: modelo explicativo. Terapia psicológica, 36(3), 156-166.

Derogatis, L. (1994). SCL-90-R. Symtomp Checklist-90-R. Administration, Scoring and Procedures Manual (ed.) Minneapolis: National Computer Systems.

Fisher, H. (2005). Porqué amamos. Naturaleza y química del amor romántico. México: Santillana. 
García, D. (2014). Narración del duelo en la ruptura amorosa. Revista Electrónica AJAYU (RAP), 12(2), 288-307.

Goicoechea, P. (2018). El amor y el desamor en la adolescencia. Revista de Formación Continuada de la Sociedad Española de Medicina de la Adolescencia, 6(2), 78-84.

González, F. (2016). Narrativas de la experiencia de mujeres mexicanas de la ciudad de Guadalajara Jalisco en el proceso de duelo por divorcio. Revista de Psicoterapia Relaciona e Intervenciones Sociales, 34, 47-40.

Hernández, R., Fernández, C., \& Baptista, P. (2010). Metodología de la investigación. México: McGraw-Hill.

Lemos, M., \& Londoño, N. (2006). Construcción y validación del cuestionario de dependencia emocional en población colombiana. Acta Colombiana de Psicología, 9(2), 127-140.

Lemos, M., Londoño, N., \& Zapata, J. (2007). Distorsiones cognitivas en personas con dependencia emocional. Informes psicológicos, 9(9), 55-69.

Keller, M., \& Nesse, R. (2005). Is low mood an adaptation? Evidence for subtypes with symptoms that match precipitants. Journal of Affective Disorders, 86(1), 27-35.

Laca, F., \& Mejía, J. (2017). Dependencia emocional, consciencia del presente y estilos de comunicación en situaciones de conflicto con la pareja. Enseñanza e Investigación en Psicología, 22(1), 66-75.

Locker, L., Mclntosh, W., Hackney, A., Wilson, J., \& Wiegand, K. (2010). The Breakup of Romantic Relationships: Situational
Predictors of Perception of Recovery. North American Journal of Psychology, 12(3) 565-578.

Márquez, M., Del Castillo, C., \& Balderrama, J. (2017). Characterization of a breakup in couples of university students: Differences in cognitive assessment of the event. Journal of Behavior, Health \& Social Issues, 9(2), 99-104.

Plunkett, C. (2016). When We End, Where Do I Begin? Exploring The Impact Of Relationship Dissolution on Self-Concept Among African-American Women. (Tesis doctoral). University Of Oklahoma, Norman, Oklahoma.

Rocha, B., Avendaño, C., Barrios, M., \& Polo, A. (2017). Actitudes hacia el amor en relaciones románticas de jóvenes universitarios. Praxis \& Saber, 8(16), 155-178. doi: 10.19053/22160159. v8.n16.2017.4596

Sánchez-Aragón, R., \& Retana-Franco, B. (2013). Evaluación tridimensional del duelo amoroso en México. Revista Iberoamericana de Diagnóstico y Evaluación-e Avaliação Psicológica, 36(2), 49-69.

Sierra, V., Rodríguez, J. \& Torres, C. (2017). Incidencia del duelo en la ruptura amorosa en estudiantes universitarios en un Centro de Crisis, Emergencias y Atención al Suicidio (CREAS). Journal of Behavior, Health \& Social Issues, 9(2), 27-35. doi:10.1016/j.jbhsi.2018.01.001

Tashiro, T., \& Frazier, P. (2003). "I'll never be in a relationship like that again": Personal growth following romantic relationship breakups. Personal Relationships, 10(1), 113-128. 
Urbiola, I. \& Estévez, A. (2015). Dependencia emocional y esquemas desadaptativos tempranos en el noviazgo de adolescentes y jóvenes. Psicología Conductual, 23(3), 571-587.

Uribe, D., \& Vélez, M. (2016). La comprensión del duelo desde la psicoterapia humanista. Revista Electrónica Psyconex, 8(12), 1-9.
Zapata, B., \& Gutiérrez, I. (2016). Salud sexual y reproductiva. Almería. España: Editorial Universidad Almería. 